\title{
Enhancing Road Safety in the MENA Region Using a Big Data App
}

\author{
Driss Kettani \\ Al Akhawayn University in Ifrane \\ Morocco
}

\begin{abstract}
This paper describes a Big Data Platform that we developed in order to fulfill the needs of an international development project we recently were involved in. The focus is on the technical aspects in terms of design and architecture of this platform, as well as on particular decisions/orientations we made to accommodate the constraints characterizing Big Data Systems.
\end{abstract}

Keywords- Big Data, MENA Region, Transport sector, Accidents Black Spots.

\section{General Context}

I recently was involved in an interesting Project titled 'Harnessing the Economic Power of Data in the Middle East and North Africa', funded by the International Development Research Centre of Canada (www.idrc.ca). The goal of this project $^{1}$ was to explore the potential of Big Data Driven Innovations to improve data collection, management, and usability, particularly in the areas of Entrepreneurship and youth employment in the Middle East and North Africa (MENA) region.

The specific objectives of this 2-year Project were to: Inform policies and regulations to effectively produce, manage, and use data, particularly for entrepreneurship and youth employment ; Study, develop, and test innovative approaches to produce, manage, and use data; Build capacities in data production, management, and use among policymakers, statistical agencies, and youth; Disseminate knowledge and lessons learned from the project; And engage key stakeholders, including, entrepreneurs, researchers, and the public.

As a partner in this project, along with colleagues from the American University in Cairo and Birzeit University in Palestine, I was tasked to develop a platform to experiment and enable the new usage/exploitation models and semaphores proposed by the research team, and to elaborate an innovative data model, which takes into account the limitations and challenges of the MENA Region.

In this paper, we particularly focus on the technical aspects in terms of design and architecture of this platform, as well as on the particular decisions/orientations we made to accommodate the constraints related to Big Data.

See https://www.idrc.ca/en/project/harnessing-economic-power-datamiddle-east-and-north-africa, for more details

\section{Why an e-Transport App?}

The transport sector is central to the development targets of MENA countries and crucial in the acceleration of their economic growth. It is also key to regional integration and vital to improving the quality of life and reducing poverty. However, most countries in the MENA region are suffering serious problems related to road accidents leading to more than 75,000 causalities every year [5]. In terms of fatalities per 10,000 registered motor vehicles, the MENA region's average rate 189 of road crash fatalities is currently one of the highest in the world. And, worst of all, in several countries of this region, an average of one person is killed in road crashes every hour [5]. It is noticeable that accidents frequently happen in the same location and for the same reasons (bad infrastructures, road conditions, heavy traffic, Speed, signalization, etc.), leading to black spots that continuously trap new victims although they are known to local/central officials [5]. This is a "Pattern" that has been highlighted and documented in many countries, along with many other patterns one could find in the specialized literature.

Hence the need of an application/system whose exclusive goal is to manage the Accidents Black Spots (ABS), through an innovative technology platform. It provides real time information on key events related/associate to them, to drivers so that they can anticipate road conditions and adapt/adjust their driving behavior accordingly.

The information and warnings is delivered to drivers via their mobile phones (or any other hand-held device) using various modalities (particular rings, various volumes, vibrations, vocal indications, video illustrations, etc.) depending on the seriousness of situation.

Data related to traffic lack spots is build and consolidated with users' feedbacks and recommendations, as the application is being used. The System also creates a depository of drivers' testimonies to be consulted by other drivers who want to plan their trip prior to hitting the road, and prepare for it with tips, landmarks, particular information, etc.

We believe such an application will enhance drivers' awareness and knowledge of road conditions and imminent risks. It would particularly contribute to:

- Decreasing the number of accidents in general;

- Deceasing fatalities and injuries and associated economic and social cost;

- Fixing/repairing black spots; 
- Building a national data repository related to road accidents and Black Sports ;

- Increasing available traffic data for decision makers to better use the available resources.

\section{Design Issues}

\section{A. Big Data or not?}

Based on several widely accepted Big Data [2] \& [3], we can safely confirm that ABS is a Big Data System since it fulfills most of the required features:

- Volume and Variability of Data : the potential number of ABS users and the nature of documents that are included in the data repository in order to serve as evidence of blackspots, result in a very high volume of data (in the Petabyte range) in different formats (GIS, audio, video, text, narration, etc.);

- Velocity: the combination of the high numbers of accidents / users / blackspots necessarily leads to a high number of database access and associated data/information updates;

- Variety of Sources: ABS system accepts multiple sources of data to build/consolidate its database, ranging from mobile phones and hand-held devices to direct access through voice recorders, cameras and any other USB input devices. Remote access, by system administrators, is also allowed through wired or wireless connections.

- Veracity of Data: ABS platform is mainly based on Data directly coming from end users, (testimonies, comments, pictures, etc.), the issue of truth/veracity of Data is crucial to address. Who is going to record new/old events and how? Would simple users/drivers be officially allowed to create new records? How to validate these records?

\section{B. System Architecture}

Currently, the n-Tiers Architecture is the most popular architecture for conventional Business applications ${ }^{2}$. Each system component (Data, Processes, Presentation/User Interface) is developed within a totally dedicated environment (tier) in terms of software, hardware, Operating System and IT Governance. When necessary, and depending on the business work flows, tiers communicate with each other through standard local or remote communication protocols and procedures.

In our case, due to the constraints and particular features associated to Big Data Systems (data amount could reach/exceed the Petabyte range, data is stored in

\footnotetext{
See http://www.codeproject.com/Articles/430014/N-TierArchitecture-and-Tips, for more details
}

different/unknown structures/formats, high velocity, etc.), the conventional n-Tiers architecture could not fulfill all of our specifications. For these reasons, the different programming relations between Data and Processes (who, where, when, etc.) needed to be rethought so that instead of having Data located in a server and Processes accessing them remotely for the purpose of business requests, we stored data in several (data) Servers and, upon users requests', it is "streamed" over Processes Servers to be exploited.

Figure 1 illustrates a Big Data System Architecture which inspired us for our system.

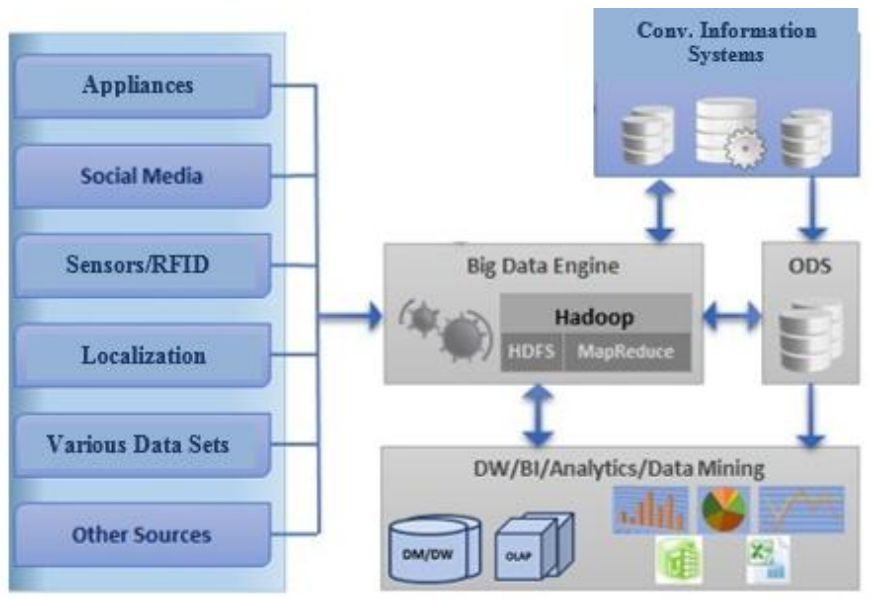

Figure 1. Conceptual Architecture of Big Data [4]

This Architecture supports various sources of Data including Governmental Data, Social Media Data, Sensors, and other Structured or Unstructured Sources. The Big Data Engine is capable of processing large volumes of data ranging from a few Megabytes to Petabytes of data of different varieties, structured or unstructured, coming in at different speeds and/or rates.

It is important to understand that the Big Data Engine is strictly tight to the Big Data Depository, and its main goal which is to process Big Data and extract from it valuable insights and/or Information for the Business (analytics). Hence the need of a simple Querying Language and Visualization tools to allow an effective interaction with data analysts.

Some of the outputs produced by the Big Data Engine could be stored in the conventional Database (official statistics and records, government reports, etc.) of the organization (ministry of transportation for instance), and becomes an integral part of it. The rest will feed specific data analysts needs' and might or might not be stored for a long term.

The Technology stack that we have used to develop the ABS system consists of:

- $\quad$ Android Studio as IDE (with JAVA as a programming language) ;

- SQLite as a client-side's DBMS ;

- MySQL as a server-side's DBMS ; 
- Google MAPS API, for GIS related functions programming ;

- Android as a client side OS Linux as a server-Side OS. 2:

The ABS system comprises 5 modules as shown in figure

- RESTful Web Service: an Object-Oriented PHP built API that reads/writes from a MySQL database, both hosted on a shared Linux web server running Apache.

- Web Admin Interface: a web interface built using HTML/CSS/jQuery/PHP that consumes the RESTful API by allowing administrators to manage users, events and their categories.

- Mobile Application: an Android application that consumes the RESTful API as well by allowing users to view and publish events.

- Google Maps Android API: this API is consumed by the mobile application to display maps to the users. These maps are customized using a custom JSON Map Style and custom markers.

- Firebase Cloud Messaging: the system relies on this messaging platform to monitor the application, manage analytics and deliver real-time notifications to the users.

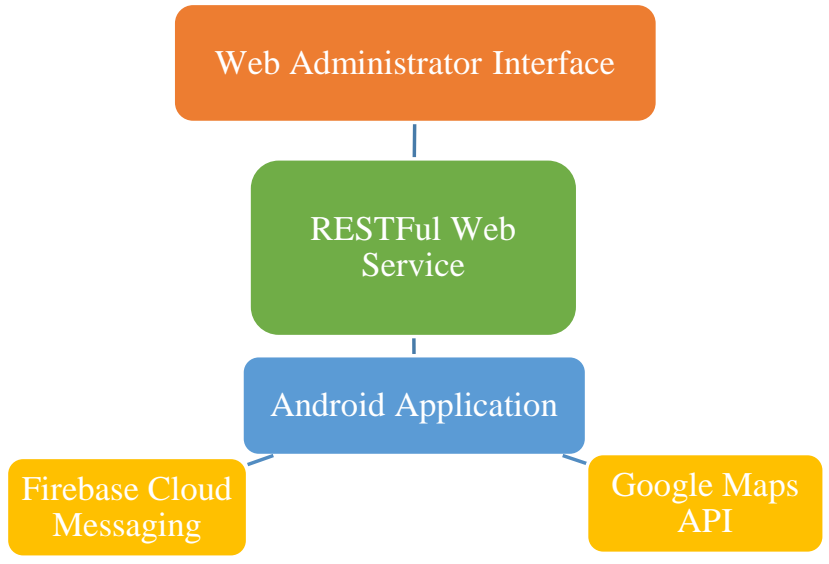

Figure 2. ABS System Architecture

\section{System Users}

ABS manages 4 main categories of users:

- System Administrators: through the web administrator interface, administrators have the privileges to manage all aspects related to Users, Categories, Events, and the system security;
- Normal users: these users have downloaded the mobile application and can receive notifications about the events (black spots) being published around them.

- Subscribed users: these users downloaded and subscribed to the mobile application using their Facebook or Google accounts. Subscribed users can add events, which require the administrator's approval before being published to the mobile application;

- Premium users: this category of users can publish events (commercial events such as publicity at a rest area along a highway) directly to the mobile application without requiring the administrator's approval.

\section{Data collection}

One important feature of ABS is to collect and put together Data related to black spots. Users at large can use the system at their front end to add new events (a black spot, an accident, work in progress, etc.) or to modify existing ones (see figure 3).

A variety of data sources is supported by the system to accommodate different data formats (text, voice, video, pictures, etc.) and different input devices (handheld devices, USB plug and play, etc.).

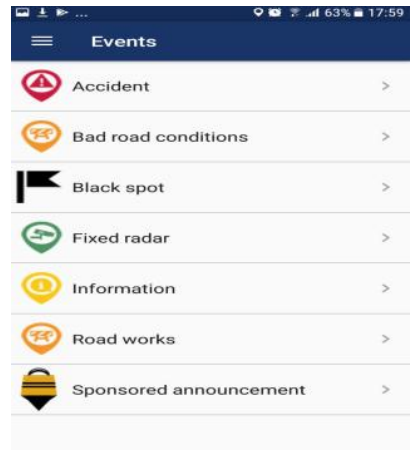

Figure 3. Interface to notify/modify events

For each user, various data is collected at the back office. These are the itineraries established, time stamped positions along the itineraries, the events published, and the notifications received, etc.

So far, only a limited sample of black spots and other related traffic events have been entered by our research team to test the platform. Indeed, the real potential of the system cannot be reached until a significant effort is made to enter a massive quantity of data at the national/MENA levels. Figure 4 shows the location of the events sample that are included so far in the ABS database. 


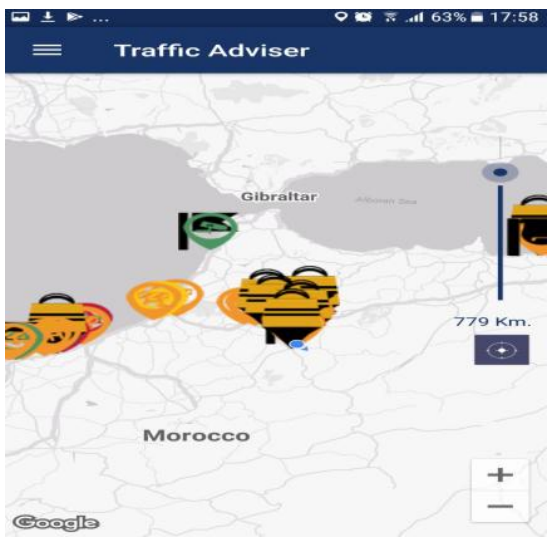

Figure 4. Location of the ABS events sample

In order to fetch nearby black-spots, a lookup radius is established and calculated using a function of the user's current speed and a time Hence, a location service is started as soon as the user launches the trip mode. This service takes care of getting the current position and speed of the user, writing them it to the database, fetching the nearby events and sending notifications about them.

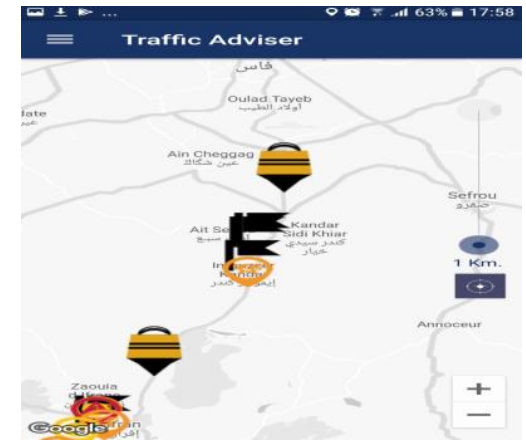

Figure 5. A focus on Morocco National Road RN.8

As the user/driver gets close to a particular black-spot, the system issue warnings in different formats and different modalities (figure 6).

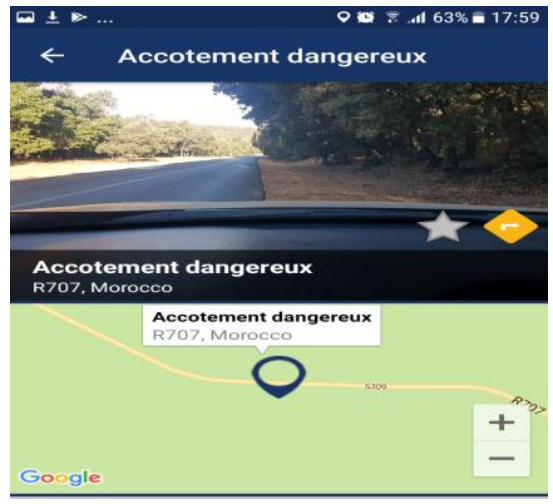

Figure 6. An example of ABS user warning

\section{Conclusion}

We presented in this paper a Big Data platform that we developed in the framework of a broader international development research project. This platform aims at informing drivers about accidents black spots that are frequent in the MENA Region, and daily cause causalities and injuries. There is no doubt, giving the alarming road security records in this region, that such a system is of benefits and has a huge potential to enhance the situation.

We first demonstrated that our platform actually fulfills all characterizing features of Big Data Systems (volume, velocity, variety, etc.) and provided a general architecture that inspired our own. Then we described the design of our system along with the technology enablers that we have used. We finally presented some user interface snapshots and their associate functionalities.

At first glance, one might legitimately argue that there is nothing particular with our system since a lot of similar systems, both licensed and free/open, exist in the market and offer about the same functionalities. However, our system is exclusively dedicated to manage accidents black spots, which is a very local issue that needs "localized" data and knowledge, locally produced, customized and packaged for the end user.

Big Data Technology is still very embryonic in the MENA region and, aside applications for home land security and defense, we were not able to identify a single good example of usage in the whole region! That clearly shows the originality and the potential of our system and of the broader research project in general. We think that there is no chance, at least in the middle run, that the Big Brothers (Google, Facebook, etc.) invest in such adventure due to the complexity of the paratechnology environment (social, politic, cultural, etc.) and the difficulty of elaborating a viable business model (that generates a cash profit!). We are still exploring in our research team ways to efficiently and cost effectively disseminate the ABS app as largely as possible, and elaborating for it an appropriate business model.

\section{Acknowledgment}

This is to express my appreciation and gratitude to the International Development Research Centre (IDRC) of Canada for funding this Project. Without such assistance this Research could not have been achieved.

\section{References}

[1] Beyer L.A and Laney D., "The Importance of Big Data: A Definition", Stamford, CT: Gartner, 2012.

[2] Habibzadeh, F., "Traffic Collisions in the Middle East", The Lancet, Vol. 380, Elsevier 519 Ltd., November 2012.

[3] International Business Machines (IBM), "What is Big Data? - Bringing Big Data to the Enterprise", http://www-01.ibm.com/software/data/ bigdata/, July 2013.

[4] Kettani, D., "Towards a Framework for Executives and Decision Makers to Discriminate Big Data Projects for International Development", IJOER, Volume-2, Issue 5, 2016. 
Proc. of The Seventh International Conference on Advances in Computing, Electronics and Communication - ACEC 2018 Copyright $\odot$ Institute of Research Engineers and Doctors, USA. All rights reserved.

ISBN: 978-1-63248-157-3 doi: 10.15224/978-1-63248-157-3-01

[5] Naji, J.A., and Djebarni, R., "Shortcomings in Road Accident Data in Developing Countries, Association of Traffic and Safety Sciences, Vol. 24, No. 2, Elsevier, 2000 